\title{
A non-iterative linear retrieval for infrared high-resolution limb sounders
}

\author{
L. Millán ${ }^{1, *}$ and A. Dudhia ${ }^{1}$ \\ ${ }^{1}$ Atmospheric, Oceanic and Planetary Physics, Clarendon Laboratory, Department of Physics, Parks Road, Oxford, UK \\ *now at: Jet Propulsion Laboratory, California Institute of Technology, Pasadena, California, USA
}

Correspondence to: L. Millán (luis.f.millan@jpl.nasa.gov)

Received: 21 December 2012 - Published in Atmos. Meas. Tech. Discuss.: 22 January 2013

Revised: 23 April 2013 - Accepted: 24 April 2013 - Published: 23 May 2013

\begin{abstract}
Currently, most of the high-spectral-resolution infrared limb sounders use subsets of the recorded spectra (microwindows) in their retrieval schemes to reduce the computing time of rerunning the radiative transfer model. A fast linear retrieval scheme is described which allows the whole spectral signature of the target molecule to be used. We determine that pressure and temperature retrievals can be treated linearly up to a $20 \%$ difference between the atmospheric state and the linearisation point for a $3 \%$ error margin and up to $10 \mathrm{~K}$ "difference" for a $3 \mathrm{~K}$ error margin near the stratopause and less than $0.5 \mathrm{~K}$ elsewhere. Assuming perfect $p T$ knowledge, $\mathrm{CH}_{4}$ retrievals can be be treated linearly up to a $20 \% \mathrm{CH}_{4}$ concentration "difference" for a $2 \%$ error margin. As an example, this technique is implemented for the Michelson Interferometer for Passive Atmospheric Sounding instrument, but it is applicable to any high-resolution limb sounder.
\end{abstract}

\section{Introduction}

In an infrared limb-viewing instrument, the observed radiance is a function of the wavenumber, pressure, temperature and composition along the viewing path (the atmospheric profile). Hence, a set of measurements at different viewing angles (different tangent heights) may be used to infer geophysical parameters of the scene against altitude (Gille, 1971). The inversion of such atmospheric radiances into atmospheric parameters is basically the problem of fitting a theoretical model (the forward model) to observations subject to errors.
The forward model simulates the observations using the radiative transfer equation. It assumes a given atmospheric state to estimate the radiance that reaches the instrument and then modifies it to consider the instrumental effects such as finite field of view (FOV) and the instrument spectral response. The retrieval procedure is the search of the atmospheric state whose simulated radiances best fit these observations.

A common approach used to find such an atmospheric state is the least-squares fit or a constrained version of it. The core of these approaches is to linearise the relationship between the measurements and a given atmospheric state. However, the observations do not depend linearly on the atmospheric state and hence the process needs to be iterated. At each iteration the forward model is run using the atmospheric estimate from the previous iteration as a linearisation point, and the atmospheric estimate is corrected according to a recipe (e.g. minimisation of least squares) until the given convergence criteria are fulfilled.

High-spectral-resolution limb sounders, such as the Michelson Interferometer for Passive Atmospheric Sounding (MIPAS) (Endemann, 1999; Fischer, 2000; Fischer et al., 2008) and the Atmospheric Chemistry Experiment (ACE) (Bernath et al., 2006), provide thousands of spectral measurements each scan, increasing dramatically the computing time spent by the retrieval running the forward model. To reduce this computing time, most retrieval schemes select optimum subsets of spectra (microwindows) that contain most of the potential information (Dudhia et al., 2002). However, even using this microwindow approach, when the signal-to-noise $(\mathrm{S} / \mathrm{N})$ ratio is small the number of microwindows needed to process in order to obtain a useful signal is 
too computationally expensive to include in an online algorithm.

This study investigates how close the linearisation point needs to be to the solution in order to be able to perform a linear (non-iterative) retrieval scheme (i.e. to establish a linear regime for the retrievals). That is to say, to only perform the last iteration step in the iterative schemes. An inversion under these conditions can use precomputed radiative transfer calculations allowing the incorporation of all the spectral profiles corresponding to the target parameters without worrying about the computing speed limitations associated with rerunning the forward model.

We examine how close climatological zonal mean profiles come to satisfying this linear range. Also, we suggest a method for linearising the propagation of temperature and pressure effects on the gas concentration retrievals.

\section{Inverse model}

The task of the inverse problem is to estimate an atmospheric profile $\boldsymbol{x}$ that best matches the measured radiances $\boldsymbol{y}$. A common solution used is to use a least-squares fit - in other words, to minimise the sum of the square differences between the vector $\boldsymbol{y}$ and the simulated measurements $\mathbf{F}\left(\boldsymbol{x}_{i}\right)$ around a given linearisation point $\boldsymbol{x}_{i}$.

The iterative Twomey-Tikhonov regularised (Tikhonov, 1963; Twomey, 1963) least-squares-fit solution is given by (von Clarmann et al., 2003)

$$
\begin{aligned}
\boldsymbol{x}_{i+1} & =\boldsymbol{x}_{i}+\left(\mathbf{K}_{i}^{T} \mathbf{S}_{y}^{-1} \mathbf{K}_{i}+\gamma^{-1} \mathbf{R}\right)^{-1} \\
& {\left[\mathbf{K}_{i}^{T} \mathbf{S}_{y}^{-1}\left(\boldsymbol{y}-\mathbf{F}\left(\boldsymbol{x}_{i}\right)\right)-\gamma^{-1} \mathbf{R}\left(\boldsymbol{x}_{i}-\boldsymbol{x}_{a}\right)\right] }
\end{aligned}
$$

where $\mathbf{K}_{i}$ is the Jacobian matrix $\left(K_{j k}=\partial y_{j} / \partial x_{k}\right)$ evaluated at iteration $i$ using the forward model around the atmospheric profile $\boldsymbol{x}_{i}, \mathbf{S}_{y}$ is the measurement error covariance matrix, $\boldsymbol{x}_{a}$ is the related a priori profile, $\gamma$ is a factor that determines the strength of the regularisation, and where $\mathbf{R}$ is a regularisation matrix which can be chosen to constrain the magnitude of a given order derivative of the retrieved profile $\boldsymbol{x}_{i}$. This technique estimates the uncertainties in the retrieved state vector $\boldsymbol{x}$ according to

$\mathbf{S}_{x}=\left(\mathbf{K}^{T} \mathbf{S}_{y}^{-1} \mathbf{K}+\gamma^{-1} \mathbf{R}\right)^{-1} \times \mathbf{K}^{T} \mathbf{S}_{y}^{-1} \mathbf{K}\left(\mathbf{K}^{T} \mathbf{S}_{y}^{-1} \mathbf{K}+\gamma^{-1} \mathbf{R}\right)^{-1}$

where $\mathbf{S}_{x}$ is the covariance matrix of the estimated atmospheric profile $\boldsymbol{x}_{i+1}$.

If the linearisation point is sufficiently close to the solution, it follows that the solution may be obtained in one iteration using $\boldsymbol{x}_{0}$ (usually called initial guess) and precomputed Jacobians $\mathbf{K}_{0}$, hence requiring no online forward model calculations. The first task is to establish the "distance" $\boldsymbol{x}-\boldsymbol{x}_{0}$ over which the following equation applies:

$\boldsymbol{y}-\mathbf{F}\left(\boldsymbol{x}_{0}\right) \simeq \mathbf{K}_{0}\left(\boldsymbol{x}-\boldsymbol{x}_{0}\right)$.
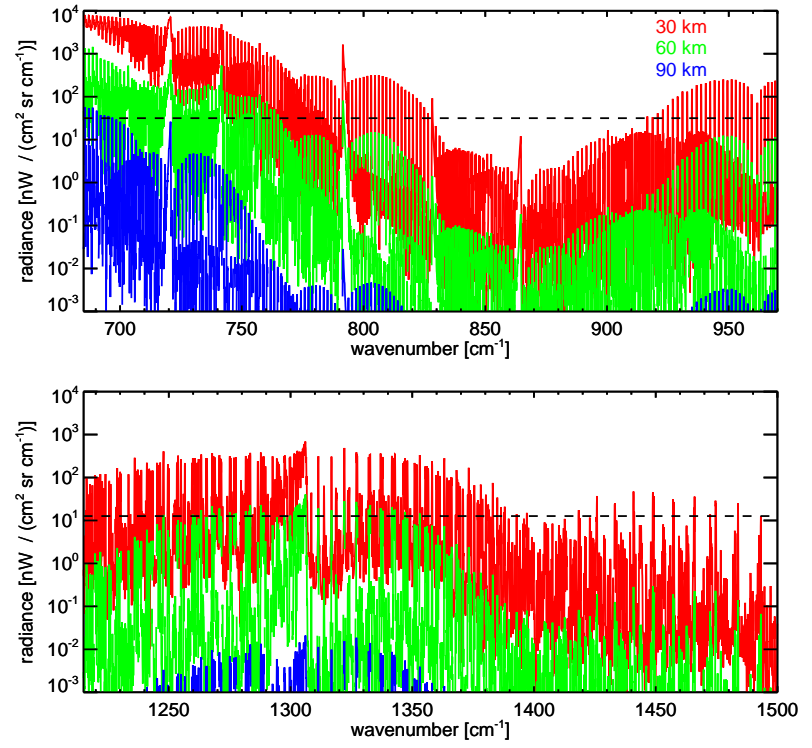

Fig. 1. (top) $\mathrm{CO}_{2}$ spectra for midlatitude conditions. (bottom) $\mathrm{CH}_{4}$ spectra for midlatitude conditions. The dashed black line shows the typical MIPAS noise equivalent spectral radiance (NESR) value.

\section{Linear regimes}

Simulations of $\mathrm{CO}_{2}$ and $\mathrm{CH}_{4}$ spectra were used to investigate how close to the solution an initial guess profile needs to be in order to lie within the linear regime. Then the question of whether the atmospheric state can be predicted within this linear regime was investigated.

As an example of the $\mathrm{CO}_{2}$ and $\mathrm{CH}_{4}$ spectra used, Fig. 1 shows $\mathrm{CO}_{2}$ and $\mathrm{CH}_{4}$ modelled spectra at 30,60 and $90 \mathrm{~km}$. For $\mathrm{CO}_{2}$ these simulations were performed between the 685 to $970 \mathrm{~cm}^{-1}$ at a spectral resolution of $0.0625 \mathrm{~cm}^{-1}$ (corresponding to the MIPAS band A spectral range for the optimised resolution data; for more information see Sect. 7). For $\mathrm{CH}_{4}$ these simulations were performed between 1215 and $1500 \mathrm{~cm}^{-1}$ (MIPAS band B).

In this study the forward model used is the reference forward model (RFM) (Dudhia, 2012a).

To test the linearity of the forward model due to pressure or temperature changes, $\mathrm{CO}_{2}$ spectra for midlatitude day conditions were simulated perturbing the entire pressure profile by $1,5,10,20$ and $50 \%$ and independently by $1,5,10,20$ and $50 \mathrm{~K}$. Then an inversion of the previously computed spectra was performed using a linear least-squares fit constrained by a Twomey-Tikhonov regularisation necessary to avoid artifacts at high altitudes induced by numerical noise inside the radiative transfer calculations.

For these simulated retrievals the spectra corresponding to the perturbed profiles were used as the measurement vector $(\boldsymbol{y})$ and the forward model and the Jacobians were computed around the midlatitude day conditions (the unperturbed scenario). Furthermore, the measurement error covariance 


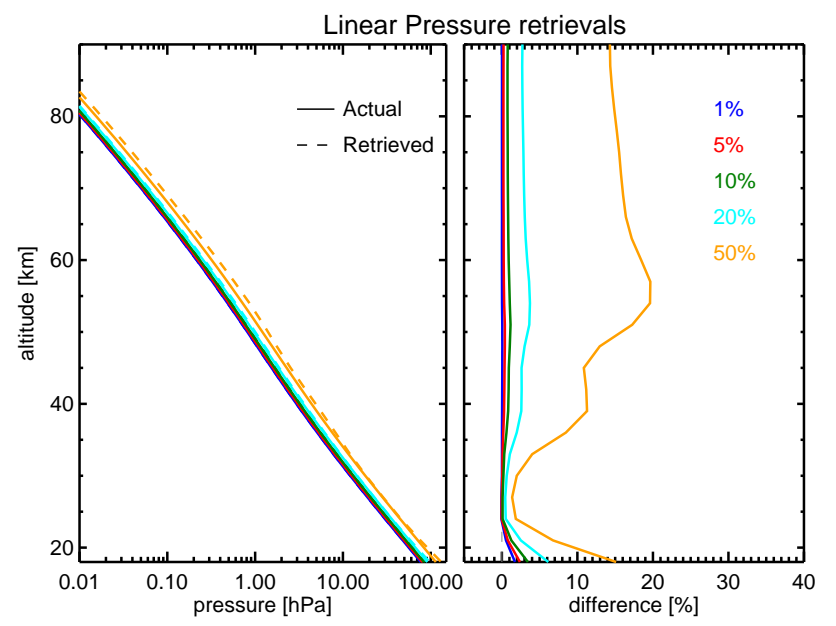

Fig. 2. Linearly retrieved pressure for different increments of the entire pressure profile (left) and percentage difference between the actual and retrieved values (right).

matrix was set to the identity matrix $\left(\mathbf{S}_{y}=\mathbf{I}\right)$. The resulting simulated retrieved values have a vertical resolution of $3 \mathrm{~km}$ in the stratosphere and lower mesosphere and around $4.5 \mathrm{~km}$ in the upper mesosphere.

As shown in Fig. 2, the linearity assumption holds up to a $20 \%$ perturbation with a $3 \%$ margin of error. In this test the entire profile was perturbed by a constant amount, while the real atmospheric perturbations will vary with altitude, but it seems a reasonable simplification.

As displayed in Fig. 3, the linearity for temperature holds up to $10 \mathrm{~K}$ perturbation with a $3 \mathrm{~K}$ error margin. However, it should be noted that this difference is localised only near the stratopause (i.e. where the temperature gradient changes sign), and outside this region the actual error margin is always less than $0.5 \mathrm{~K}$.

To try to improve the linearity of the forward model due to changes in temperature, rather than retrieving temperature directly, the Planck function or even $B_{v}(T) / T$ can be retrieved instead. For example, to retrieve temperature through the Planck function, the temperature Jacobian needs to be multiplied by $\mathrm{d} T / \mathrm{d} B_{\bar{v}}(T)$, where $\mathrm{d} T$ corresponds to a temperature change of $1 \mathrm{~K}$ and $\mathrm{d} B_{\bar{v}}(T)$ is the corresponding change in Planck function at a fixed wavenumber chosen to be in the middle of the spectral region of interest.

Figure 4 displays the retrieved and actual values for the same temperature increments as before. As can be seen the linearity of the problem improves considerably when retrieving temperature via the Planck function. However, despite this linearity improvement, this approach cannot be easily used (or the $B_{v}(T) / T$ approach) with real data because oscillations in the retrieval profile may cause negative Planck function values to be retrieved.

Once the $p T$ conditions of the scene are known, the gas concentrations may be retrieved since it is the only remaining

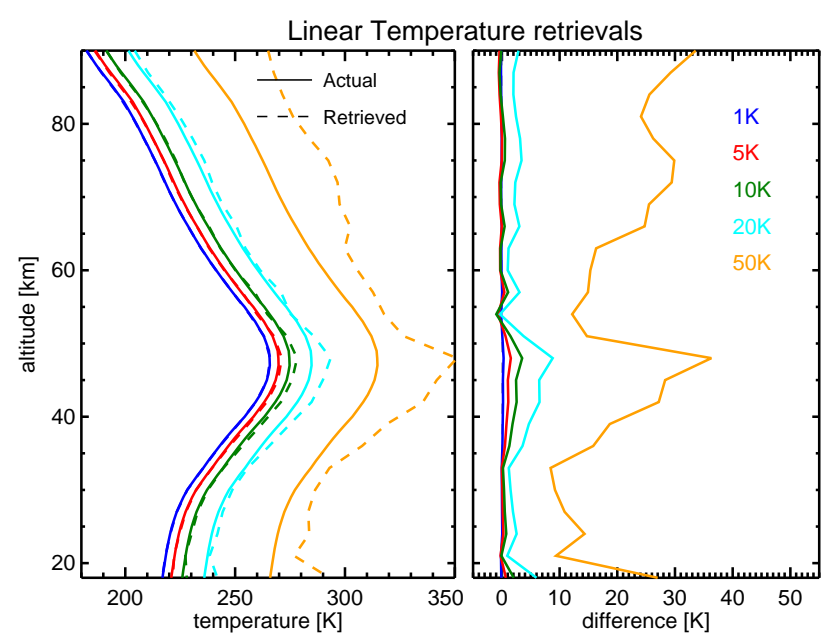

Fig. 3. Linearly retrieved temperature for different increments of the entire temperature profile (left) and absolute difference between the actual and retrieved values (right).

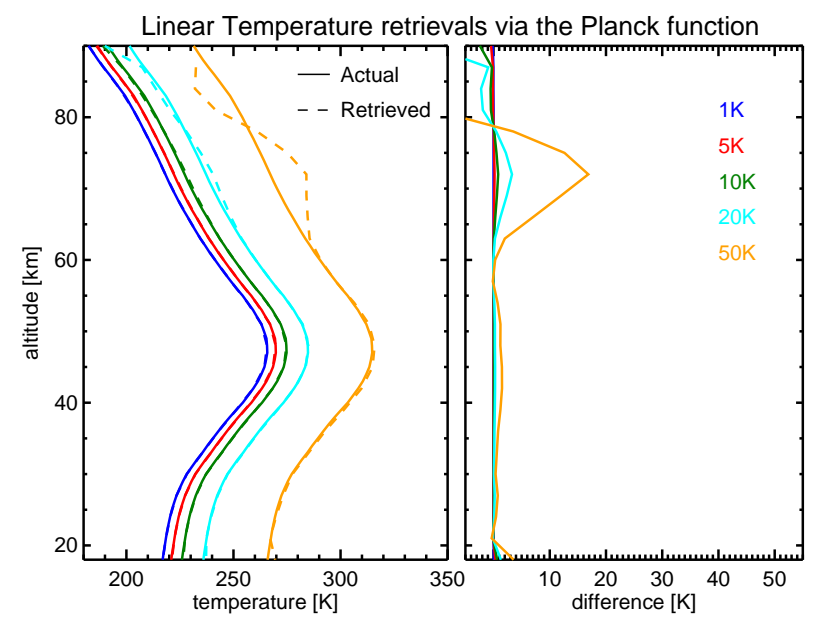

Fig. 4. Linearly retrieved temperature via the Planck function for different increments of the entire temperature profile (left) and absolute difference between the actual and retrieved values (right).

unknown in the radiative transfer model equation. Hence, the gas of interest can be retrieved selecting a spectral region where it is the principal emitter or where the emissions of the other gases are already accurately known.

First perfect knowledge of temperature and pressure will be assumed to test the linearity under ideal circumstances. Then, since the linear algorithm avoids running the forward model precomputing the $\mathrm{CH}_{4}$ simulations with the $p T$ conditions of a given linearisation point, a discussion of how to adjust these simulations to the $p T$ scene conditions (previously retrieved) will follow.

To test the linearity of the forward model due to $\mathrm{CH}_{4}$ concentration changes, $\mathrm{CH}_{4}$ spectra for midlatitudes daytime conditions were simulated perturbing the entire $\mathrm{CH}_{4}$ profile 
by $1,5,10,20$ and $50 \%$, and an inversion was performed in the same manner as before. The results are shown in Fig. 5.

As displayed, the linearity for $\mathrm{CH}_{4}$ retrievals holds up to a $20 \% \boldsymbol{x}-\boldsymbol{x}_{0}$ "distance" with a $2 \%$ margin of error at most altitudes. This linearisation range is only shown as a maximum theoretical accuracy of the linear $\mathrm{CH}_{4}$ retrieval because in practice, if the radiative transfer model is not going to be run in the retrieval scheme, the $\mathrm{CH}_{4}$ simulations will have to be adjusted for the difference between the $p T$ linearisation and scene conditions.

\subsection{Climatological variability}

Having established the linear range for a pressure, temperature and VMR retrieval, the next question is which climatological resolution is needed to ensure that the linearisation point is likely to lie within this linear range of the true state. To answer this, an analysis was performed using MIPAS middle-atmospheric retrievals from MORSE (an iterative inversion algorithm; for more information see Sect. 7.1) for the days available in June, July and August for 2007, 2008 and 2009. It is expected that high variability will be found in the Northern Hemisphere during this month.

The analysis consisted in computing the pressure, temperature or $\mathrm{CH}_{4}$ deviation from a zonal mean with respect to latitude bin mean profiles (i.e. global, $60^{\circ}, 30^{\circ}$, etc) until the deviation at any given point was smaller than the linear regime established previously. As shown in Figs. 6, 7 and 8 a global mean climatology cannot represent the variability found in the atmosphere. An analysis of these figures suggests that for pressure and temperature, a linearisation point for every $30^{\circ}$ of latitude is needed to ensure that the "distance" $\boldsymbol{x}-\boldsymbol{x}_{0}$ is less than the required $20 \%$ or $10 \mathrm{~K}$ proximity, while the $\mathrm{CH}_{4}$ retrieval needs a linearisation point for every $20^{\circ}$ of latitude to ensure that the "distance" $\boldsymbol{x}-\boldsymbol{x}_{0}$ is less than the $20 \%$ required proximity. Note that these climatology latitudinal resolutions are only tentative guidelines, they will vary with season and in particular for other trace species.

\section{Forward model adjustments}

To avoid running the radiative transfer model during the linear inversion scheme, the gas simulations are calculated using the $p T$ linearisation conditions and then adjusted to the $p T$ scene conditions retrieved in an earlier stage of the retrieval. Two adjustments are needed: one to adjust the $p T$ dependence of the simulated spectra and a second one to adjust the $p T$ dependence of the Jacobians.

\subsection{VMR forward model $p T$ adjustment}

The simulated spectra can simply be modified using the temperature and pressure Jacobian of the corresponding spectral range, i.e.

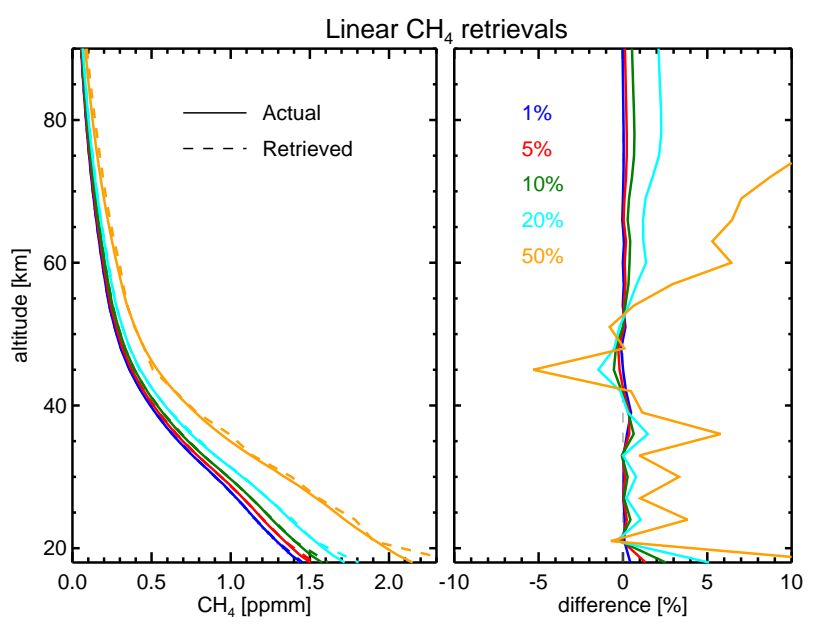

Fig. 5. Linearly retrieved $\mathrm{CH}_{4}$ for different increments of the entire $\mathrm{CH}_{4}$ profile (left) and percentage difference between the actual and retrieved values (right).

$\mathbf{F}^{*}\left(\boldsymbol{x}_{0}\right)=\mathbf{F}\left(\boldsymbol{x}_{0}\right)+\mathbf{K}_{T_{0}}\left(\boldsymbol{T}-\boldsymbol{T}_{0}\right)+\mathbf{K}_{p_{0}}\left(\boldsymbol{p}-\mathbf{p}_{0}\right)$,

where $\mathbf{F}^{*}\left(\boldsymbol{x}_{0}\right)$ is the corrected spectrum for the actual $p T$ scene conditions $(\boldsymbol{p}, \boldsymbol{T}), \mathbf{F}\left(\boldsymbol{x}_{0}\right)$ is the linearisation point simulated spectrum and $\mathbf{K}_{T_{0}}$ and $\mathbf{K}_{p_{0}}$ are the linearisation point temperature and pressure Jacobians, respectively.

Figure 9 displays the $\mathrm{CH}_{4}$ linear retrievals for different changes in temperature. To isolate the effects of the term " $\mathbf{K}_{T_{0}}\left(\boldsymbol{T}-\boldsymbol{T}_{0}\right)$ " in Eq. (4), the $\mathrm{CH}_{4}$ Jacobians used were computed with the temperature of the scene (i.e. the true temperature) with the $\mathrm{CH}_{4}$ concentration remaining constant (only the temperature varies). As shown, for a $10 \mathrm{~K}$ perturbation the errors induced are less than $\sim 20 \%$ except around $45 \mathrm{~km}$, where the deviation is almost $70 \%$. This deviation is caused by oscillations in the linear retrieval presumably induced by the $10 \mathrm{~K}$ constant difference between the linearisation profile and the atmospheric state. For a $20 \mathrm{~K}$ perturbation the retrieved values are non-realistic; for this reason the retrieved $\mathrm{CH}_{4}$ corresponding to a $50 \mathrm{~K}$ increment is not shown.

Figure 10 is the equivalent figure for pressure increments (testing only the " $\mathbf{K}_{p_{0}}\left(\boldsymbol{p}-\boldsymbol{p}_{0}\right)$ " term of Eq. 4). As portrayed, the adjustment works up to a $20 \%$ perturbation with less than $2 \%$ error margin except around 20 and $50 \mathrm{~km}$.

Figures 9 and 10 suggest that the VMR forward model simulations $p T$ adjustment given by Eq. (4) may be valid as long as the $p T \boldsymbol{x}-\boldsymbol{x}_{0}$ "distances" are not greater than $10 \mathrm{~K}$ and $20 \%$ accordingly.

\subsection{VMR Jacobians $p T$ adjustment}

As the forward model has been adjusted to the temperature and pressure of the scene, in a similar manner the VMR, $v$, Jacobians can be adjusted using

$\mathbf{K}_{v}^{*}=\mathbf{K}_{0 v}+\mathbf{H}_{T}\left(\boldsymbol{T}-\boldsymbol{T}_{0}\right)+\mathbf{H}_{p}\left(\boldsymbol{p}-\boldsymbol{p}_{0}\right)$, 

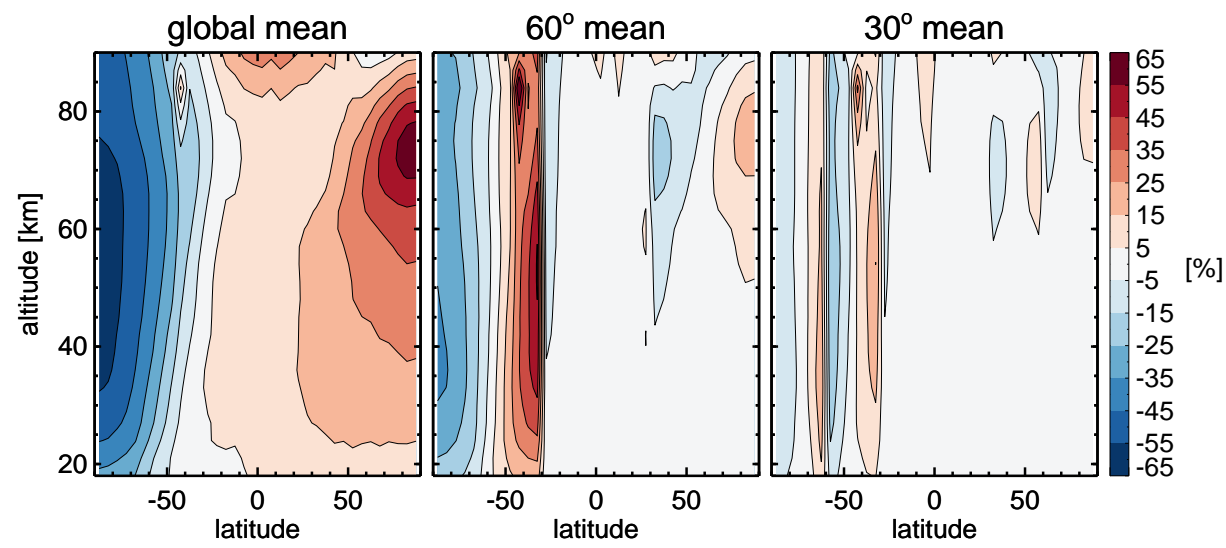

Fig. 6. Pressure variation of global (left), $60^{\circ}$ (middle) and $30^{\circ}$ (right) latitude bin profiles for the MIPAS MA mode days available in June, July and August 2007, 2008 and 2009.
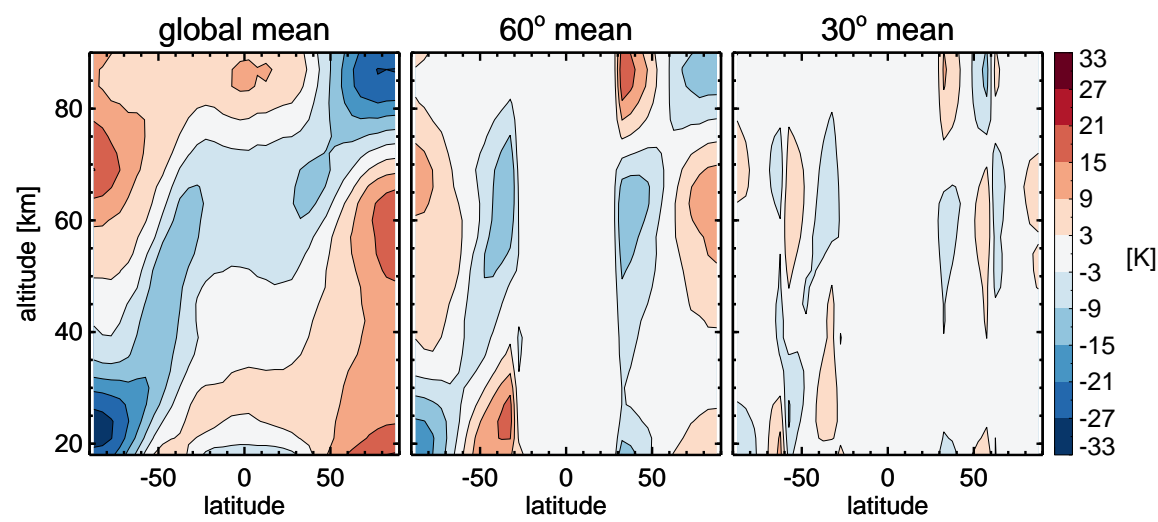

Fig. 7. Temperature variation of global (left), $60^{\circ}$ (middle) and $30^{\circ}$ (right) latitude bin profiles for the MIPAS MA mode days available in June, July and August for 2007, 2008 and 2009.

where $\mathbf{K}_{v}^{*}$ is the adjusted VMR Jacobian for the actual scene $p T$ conditions $(\boldsymbol{p}, \boldsymbol{T}), \mathbf{K}_{0 v}$ is the VMR Jacobian computed with the linearisation point conditions $\left(\boldsymbol{p}_{0}, \boldsymbol{T}_{0}\right)$, and where $\mathbf{H}$ corresponds to the changes of the VMR Jacobian with respect to either temperature or pressure; that is to say,

$H_{T i j k}=\frac{\partial K_{v_{i j}}}{\partial T_{k}}=\frac{\partial}{\partial T_{k}}\left(\frac{\partial L_{i}}{\partial v_{j}}\right)=\frac{\partial^{2} L_{i}}{\partial T_{k} \partial v_{j}}$

and

$H_{p i j k}=\frac{\partial K_{v_{i j}}}{\partial p_{k}}=\frac{\partial}{\partial p_{k}}\left(\frac{\partial L_{i}}{\partial v_{j}}\right)=\frac{\partial^{2} L_{i}}{\partial p_{k} \partial v_{j}}$,

where $i$ corresponds to the measurement tangent height, while $j$ and $k$ correspond to perturbation heights. Theoretically, these matrices are dimensionally $m \times n \times n$ (where $m$ and $n$ are the number of elements in the measurement vector $\boldsymbol{y}$ and the atmospheric profile $\boldsymbol{x}$, respectively), but it should be noted that for optically thin cases the atmospheric limb monochromatic radiance, $L_{v}$, leaving an stratified atmosphere, is given by (see appendix)
$L_{\nu} \simeq \sum_{i} B_{\nu}\left(T_{i}\right) \frac{p_{i}}{R T_{i}} l_{i}\left(\sum_{j} v_{i j} \sigma_{v(i j)}\right)$,

where $i$ refers to the $i$ th stratified level, $B(T)$ is the Planck function, $p, T$ and $l$ are the pressure, temperature and length along the given path, respectively, and where $j$ corresponds to the $j$ th absorber with absorption coefficient $\sigma$ and volume mixing ratio $v$. Equations (6) and (7) become

$\mathbf{K}_{v}=\frac{\partial L_{i}}{\partial v_{j}} \simeq B\left(T_{j}\right) \frac{p_{j}}{R T_{j}} l_{j} \sigma_{j}$,

and hence

$\frac{\partial^{2} L_{i}}{\partial T_{k} \partial v_{j}}, \frac{\partial^{2} L_{i}}{\partial p_{k} \partial v_{j}}=0 \quad$ if $\quad k \neq j$

and

$\frac{\partial^{2} L_{i}}{\partial T_{k} \partial v_{j}}, \frac{\partial^{2} L_{i}}{\partial p_{k} \partial v_{j}} \neq 0 \quad$ if $\quad k=j$,

which reduces the size of $\mathbf{H}$ to $m \times n$. 

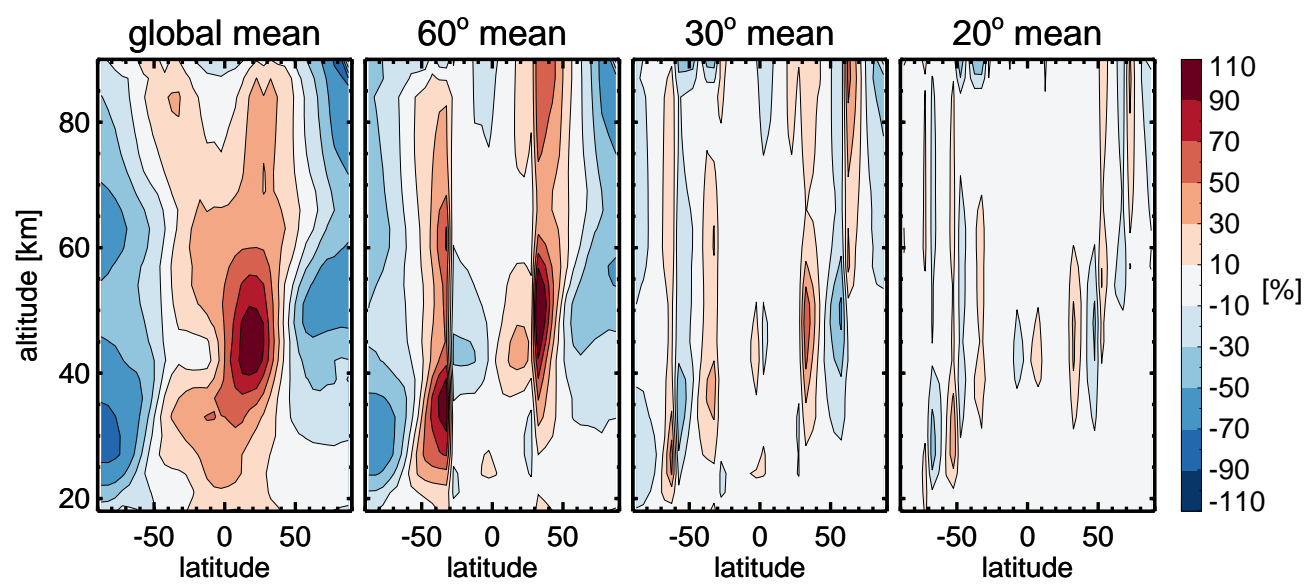

Fig. 8. $\mathrm{CH}_{4}$ variation of global, $60^{\circ}, 30^{\circ}$ and $20^{\circ}$ latitude bin profiles for the MIPAS MA mode days available in June, July and August for 2007, 2008 and 2009.

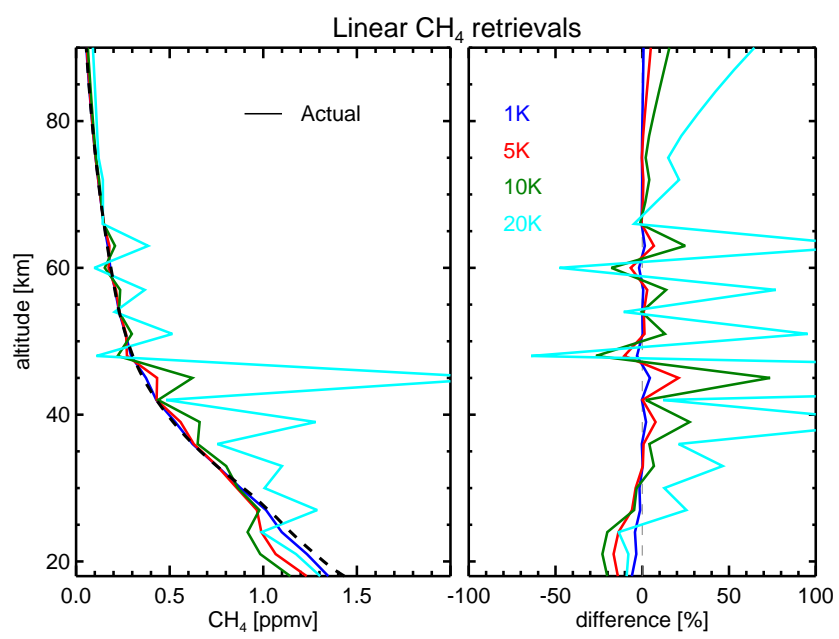

Fig. 9. Linearly retrieved $\mathrm{CH}_{4}$ concentrations (after adjusting the forward model) for different temperature increments of the entire profile (left) and percentage difference between the actual and retrieved values (right).

Although these matrices can be computed by perturbing height by height the temperature or pressure profiles and then subtracting the original VMR Jacobians as computed by the forward model, here a simpler technique was implemented. Noting that, while the VMR Jacobian at the scene conditions will be given by Eq. (9), the linearisation profile VMR Jacobian will be given by

$\mathbf{K}_{0 v}=\frac{\partial L_{0 i}}{\partial v_{j}} \simeq B\left(T_{0 j}\right) \frac{p_{0 j}}{R T_{0 j}} l_{j} \sigma_{j}$

Hence, the linearisation point VMR Jacobian can be modified to the $p T$ scene conditions by

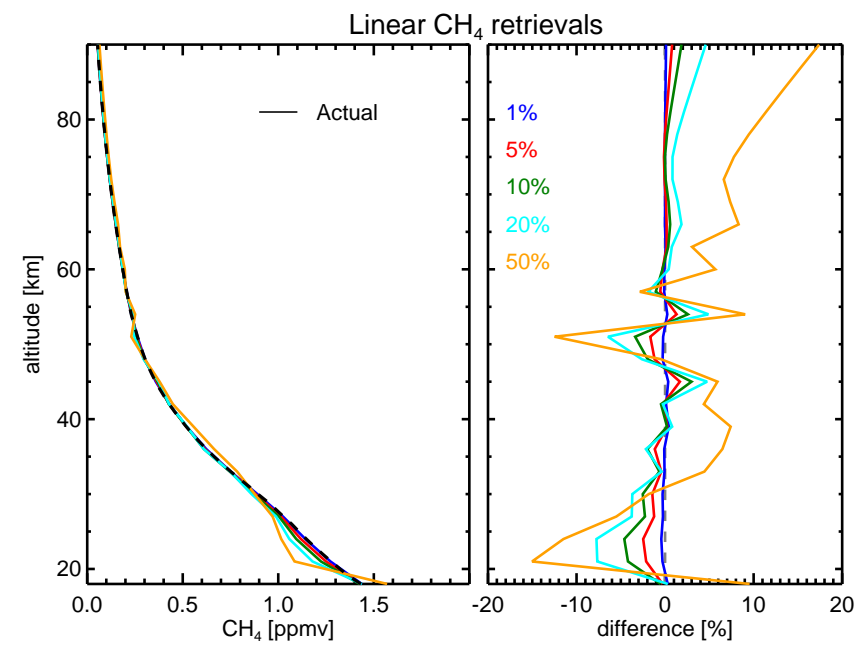

Fig. 10. Linearly retrieved $\mathrm{CH}_{4}$ concentrations (after adjusting the forward model) for different pressure increments of the entire profile (left) and percentage difference between the actual and retrieved values (right).

$\mathbf{K}_{v}^{*}=\mathbf{K}_{0 v}\left[\frac{B\left(T_{j}\right) p_{j}}{B\left(T_{0 j}\right) p_{0 j}} \frac{T_{0}}{T_{j}}\right]$.

The effect of this simple adjustment can be appreciated in Fig. 11. Note that the only parameter that it is varying in these simulations is the temperature dependence of the VMR Jacobian to isolate its effects in the retrieval. As shown when the Jacobians are not adjusted, the error induced in the retrievals for a $10 \mathrm{~K}$ perturbation are up to $30 \%$, while when the Jacobians are adjusted the errors induced are significantly reduced (less than $10 \%$ for a $20 \mathrm{~K}$ perturbation).

Figure 12 is the equivalent figure for pressure increments. As can be seen the errors induced to the retrieval when the 


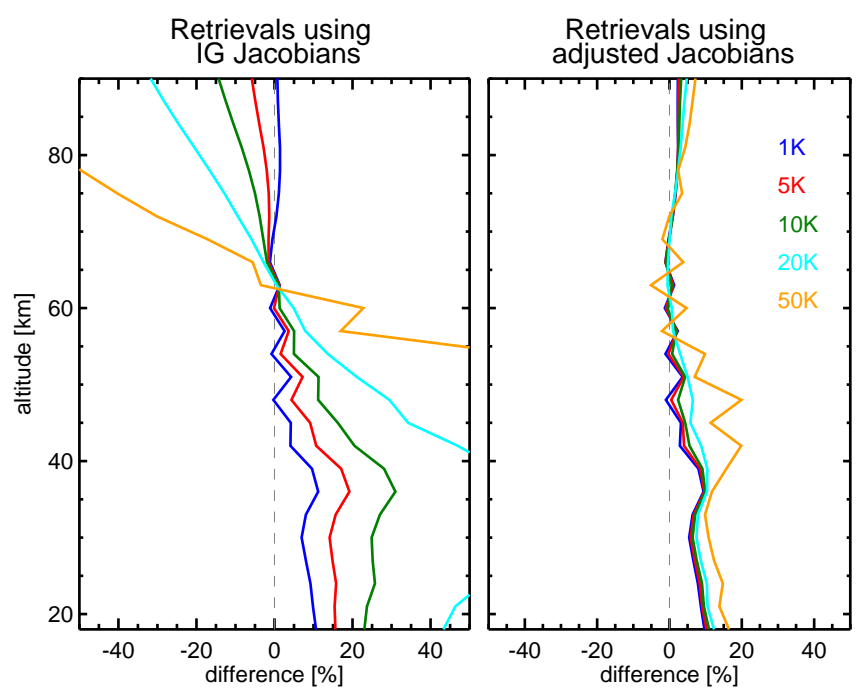

Fig. 11. Percentage difference between the actual and linearly retrieved $\mathrm{CH}_{4}$ concentrations for different increments of the entire temperature profile when using the linearisation point Jacobians (left) and when adjusting them (using Eq. 13) to the new temperature conditions (right).

VMR Jacobians are adjusted to the $p T$ conditions of the scene (right) are much smaller than when the VMR Jacobians are not adjusted (left).

\section{Linear regime summary}

The results shown so far suggest that the retrievals of pressure and temperature can be treated linearly up to a $\boldsymbol{x}-\boldsymbol{x}_{0}$ "distance" of $20 \%$ for a $3 \%$ error margin and up to a $10 \mathrm{~K}$ difference for a $3 \mathrm{~K}$ error margin near the stratopause and less than $0.5 \mathrm{~K}$ elsewhere. The $\mathrm{CH}_{4}$ simulated retrievals shown suggest that, for simulations with perfect $p T$ knowledge, the $\mathrm{CH}_{4}$ retrievals can be treated linearly up to a $20 \% \mathrm{CH}_{4} \boldsymbol{x}-\boldsymbol{x}_{0}$ "distance" with a margin of error of $2 \%$. However, since in the linear algorithm the radiative transfer model is not run, the adjustments for the $p T$ difference between the linearisation point used and the scene conditions worsen this linear error estimate. These $\mathrm{CH}_{4} p T$ adjustments hold up to a $10 \mathrm{~K}$ and $20 \%$ (pressure) $\boldsymbol{x}-\boldsymbol{x}_{0}$ "distance" with an error margin of $20 \%$ and $10 \%$, respectively.

An analysis of the pressure, temperature and $\mathrm{CH}_{4}$ variability revealed that to ensure the proximity required between the linearisation and the true profile, a climatology with a latitudinal resolution of at least $20^{\circ}$ is needed.

It should be noted that so far $p, T$ and $v$ have been treated independently, although Eq. (8) predicts that they multiply each other and that these simulations have been performed perturbing the entire profile rather than level by level, and hence these linear regimes are only a measure of guidance. Furthermore, signal-to-noise limitations, overlapping emis-

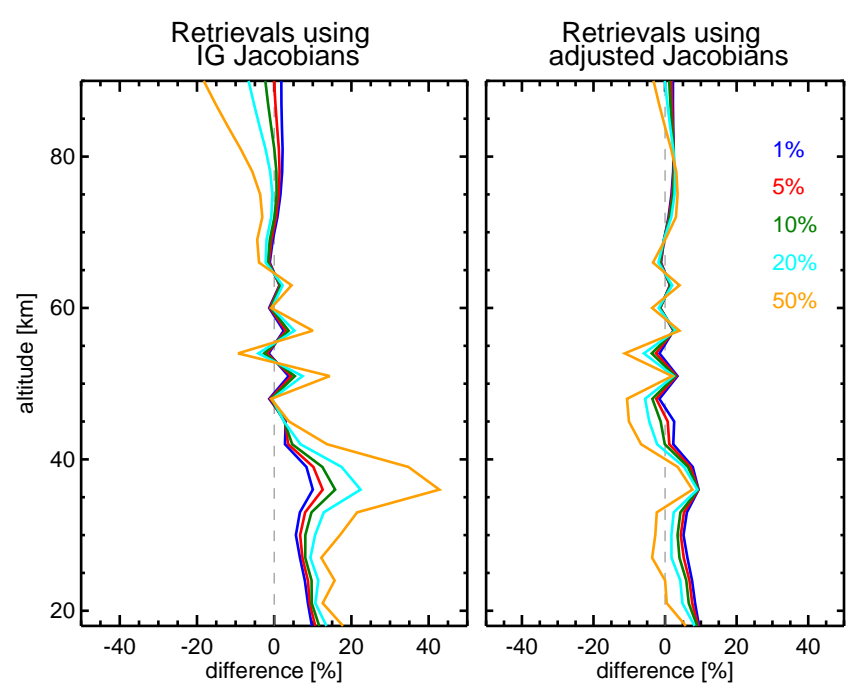

Fig. 12. Percentage difference between the actual and linearly retrieved $\mathrm{CH}_{4}$ concentrations for different increments of the entire pressure profile when using the linearisation point Jacobians (left) and when adjusting them (using Eq. 13) to the new pressure conditions (right).

sion by other molecules and nonLTE effects have been ignored. Before the linear algorithm can produce useful results in a practical application, the influence of these factors needs to be properly taken into account.

\section{Error analysis}

The total error in the linear retrieval will be given by the sum of the random, systematic and linear approximation errors. The random errors are determined by the random noise in the measurements and are given by the diagonal elements of the covariance matrix of the retrieved state (Eq. 2). The systematic errors arise from forward model uncertainties, instrumental issues and retrieval approximations and will have to be included in a full error analysis - not pursued here.

The linear approximation errors are a function of the deviation between the retrieved profile and the linearisation point. For pressure and temperature, the following equations are suggested:

$\xi_{p}=\frac{\lambda_{p}}{\Lambda_{p}}\left(\frac{\boldsymbol{p}-\boldsymbol{p}_{0}}{\boldsymbol{p}_{0}}\right)$

and

$\xi_{T}=\frac{\lambda_{T}}{\Lambda_{T}}\left(\boldsymbol{T}-\boldsymbol{T}_{0}\right)$,

where $\xi_{p}$ is the pressure approximation error in $\%$ (hence the ratio in the right-hand side of the equation) and where $\xi_{T}$ is the temperature linear approximation error in $\mathrm{K}$. Also, $\lambda$ is 
the pressure or temperature error margin (also in \% or in $\mathrm{K}$ ) established in Sect. 3 for a given perturbation, $\Lambda$.

For VMR, $v$, the linear approximation errors are given by

$$
\begin{aligned}
\xi_{v}= & {\left[\left(\frac{\lambda_{v}}{\Lambda_{v}}\left(\frac{\boldsymbol{v}-\boldsymbol{v}_{0}}{\boldsymbol{v}_{0}}\right)\right)^{2}+\left(\frac{\lambda_{v T}}{\Lambda_{v T}}\left(\boldsymbol{T}-\boldsymbol{T}_{0}\right)\right)^{2}+\right.} \\
& \left.\left(\frac{\lambda_{v p}}{\Lambda_{v p}}\left(\frac{\boldsymbol{p}-\boldsymbol{p}_{0}}{\boldsymbol{p}_{0}}\right)\right)^{2}\right]^{1 / 2}
\end{aligned}
$$

where $\xi_{v}$ is in percentage and $\lambda_{v}, \lambda_{v T}$ and $\lambda_{v p}$ are error margins in percent due to perturbations in VMR, temperature or pressure, $\Lambda_{v}, \Lambda_{v T}$ and $\Lambda_{v p}$. Note that $\Lambda_{v}$ and $\Lambda_{v p}$ are in percent and $\Lambda_{v T}$ is in kelvins.

Figure 13 displays the random error estimates of the linear pT and VMR retrievals, as well as the sum of the random errors and several linear approximation errors computed for different deviations between the retrieved profile and the linearisation point. The random errors were computed using the same spectral regions as before and assuming the characteristic noise present in the MIPAS radiances (see Sect. 7 for more details). The linear errors were computed using the error margins and perturbations discussed in Sect. 5 (for instance, $\lambda_{T}=3 \mathrm{~K}$ for a $\Lambda_{T}=10 \mathrm{~K}, \lambda_{p}=2 \%$ for a $\Lambda_{p}=20 \%$, etc.). Note that the deviations from the linearisation points were assumed constant throughout the profile and as such should be considered only as worst case scenarios.

As shown in Fig. 13, the error in the linear $p T$ or VMR retrieval, at least in the worst case scenario, when the deviation between the retrieved profile equals the maximum deviation allowed, might be too high for scientific results. In an operational retrieval many linearisation points can be tested (see Sect. 7.3) to find the one closest to the true atmospheric state, hence avoiding the maximum deviation allowed. Furthermore, if the linear approximation error is still too large, in those cases, the linear retrieval could be used as a first iteration of an iterative scheme, presumably reducing its computing time considerably.

\section{Implementation for MIPAS}

As a practical implementation, a $p T$ and $\mathrm{CH}_{4}$ linear retrieval was implemented using data from the Michelson Interferometer for Passive Atmospheric Sounding (MIPAS) instrument (Endemann, 1999; Fischer, 2000; Fischer et al., 2008) on board the Envisat satellite. MIPAS was a Fourier transform spectrometer conceived to detect limb emission spectra on a global scale. MIPAS covered the infrared spectral region between $685 \mathrm{~cm}^{-1}$ and $2410 \mathrm{~cm}^{-1}$ (divided into five bands listed in Table 1), where emission features from many atmospheric species are present.

Originally, between July 2002 and March 2004 MIPAS operated with an spectral sampling of $0.025 \mathrm{~cm}^{-1}$ (referred to as "full resolution" data); however, the instrument developed problems with the interferometer mirror slide mechanism
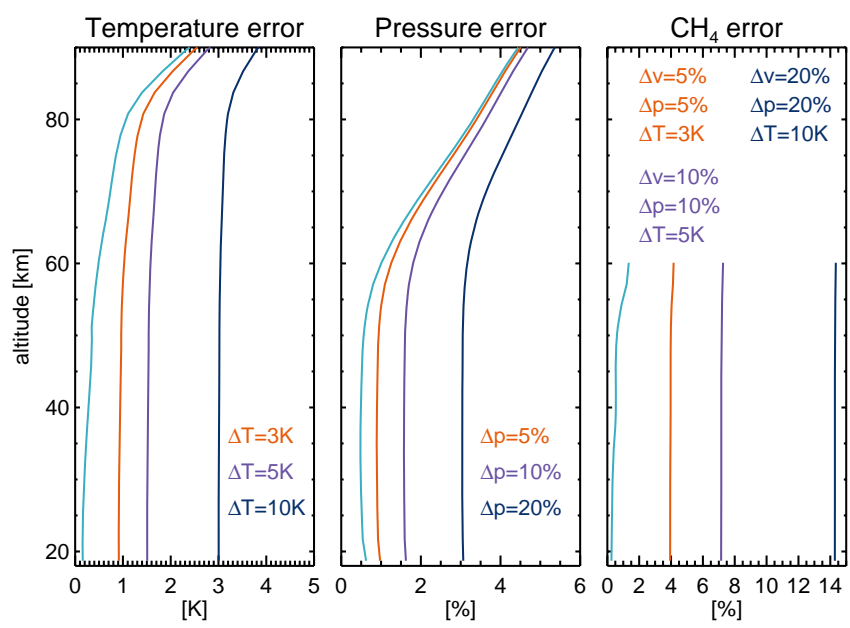

Fig. 13. Error estimates for pressure, temperature and $\mathrm{CH}_{4}$ concentration. Aqua lines show the random errors, while orange, purple and blue lines show the sum of the random errors and the linear approximation errors for different deviations between the retrieved profile and the linearisation point. The random errors correspond to estimates for midlatitude conditions, but other conditions are similar.

and the instrument was switched off. In January 2005 operations were resumed restricting the mirror movement to $40 \%$ of is original maximum displacement corresponding to a reduced spectral sampling (referred to as "optimised resolution" data) of $0.0625 \mathrm{~cm}^{-1}$. MIPAS took quasi-continuous measurements until April 2012 when the European Space Agency (ESA) lost contact with Envisat.

In the optimised resolution operation, the MIPAS nominal (NOM) measurement mode consists of 27 tangent heights from $6 \mathrm{~km}$ to $70 \mathrm{~km}$, but MIPAS altitude coverage was extended well into the thermosphere using special measurements modes (Fig. 14) such as the middle atmosphere (MA) mode with 29 tangent heights from $18 \mathrm{~km}$ to $102 \mathrm{~km}$ and the upper atmosphere (UA) mode with 35 tangent heights from $42 \mathrm{~km}$ to $172 \mathrm{~km}$. Since April 2007, MIPAS operated in MA mode for 1 day in every 10 , and similarly in the UA mode.

Here we use the spectra taken in the MA mode from band $\mathrm{A}$, where most of the $\mathrm{CO}_{2}$ lines occur, and because these $\mathrm{CO}_{2}$ lines (as opposed to the $\mathrm{CO}_{2}$ lines in band D) are close to LTE up to at least $100 \mathrm{~km}$ (López-Puertas and Taylor, 2001), and from band $\mathrm{B}$, where most of the $\mathrm{CH}_{4}$ lines occur. The MA viewing mode was selected to apply the algorithm here described because in this mode more microwindows are needed to compensate the small signal-to-noise ratio encountered at higher altitudes, and hence in this mode this algorithm has the most potential. 
Table 1. MIPAS spectral bands.

\begin{tabular}{cccl}
\hline $\begin{array}{c}\text { MIPAS } \\
\text { band }\end{array}$ & $\begin{array}{c}\text { Wavenumber range } \\
{\left[\mathrm{cm}^{-1}\right]}\end{array}$ & $\begin{array}{c}\text { NESR* } \\
{\left[\mathrm{nW} /\left(\mathrm{cm}^{2} \mathrm{srcm}^{-1}\right)\right]}\end{array}$ & $\begin{array}{l}\text { Principal atmospheric } \\
\text { emitting species }\end{array}$ \\
\hline A & $685-970$ & 32 & $\mathrm{CO}_{2}, \mathrm{O}_{3}, \mathrm{H}_{2} \mathrm{O}, \mathrm{HNO}_{3}$ \\
$\mathrm{AB}$ & $1020-1170$ & 25 & $\mathrm{CO}_{2}, \mathrm{O}_{3}, \mathrm{H}_{2} \mathrm{O}, \mathrm{N}_{2} \mathrm{O}$ \\
B & $1215-1500$ & 13 & $\mathrm{H}_{2} \mathrm{O}, \mathrm{CH}_{4}, \mathrm{~N}_{2} \mathrm{O}$ \\
C & $1570-1750$ & 4 & $\mathrm{NO}_{2}, \mathrm{H}_{2} \mathrm{O}$ \\
D & $1820-2410$ & 2.6 & $\mathrm{CO}_{2}, \mathrm{O}_{3}, \mathrm{H}_{2} \mathrm{O}, \mathrm{N}_{2} \mathrm{O}, \mathrm{NO}, \mathrm{CO}$ \\
\hline
\end{tabular}

* Noise equivalent spectral radiance (NESR) for MIPAS operating at the "optimised resolution" (Dudhia, 2007).

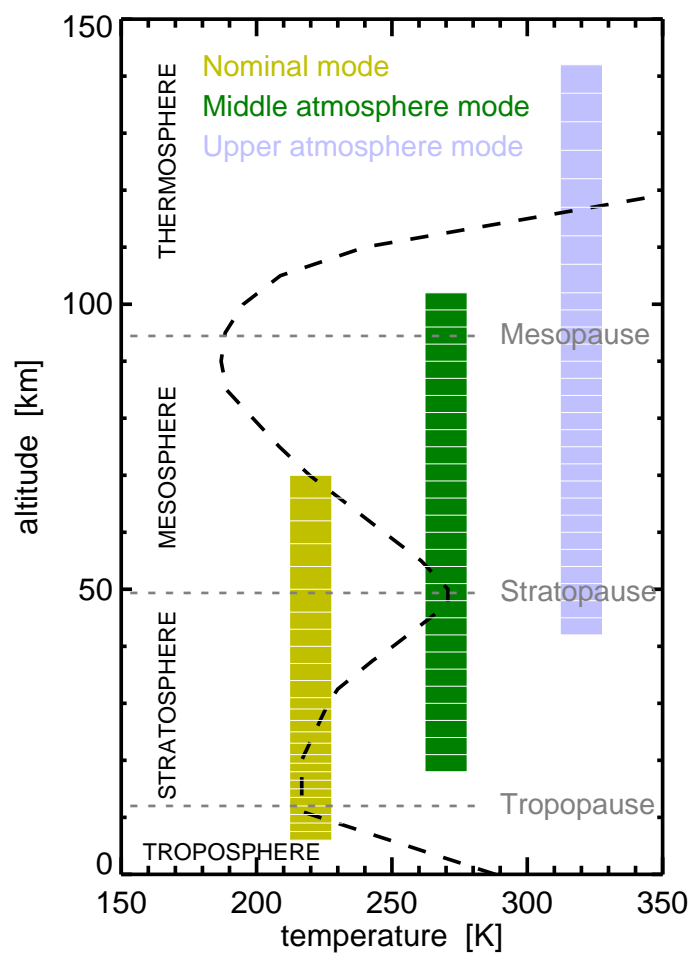

Fig. 14. MIPAS optimised resolution operation measurement modes.

\subsection{MIPAS retrieval algorithms}

Several algorithms have been developed to retrieve altitude profiles of temperature, pressure and composition from the MIPAS spectra (Ridolfi et al., 2000; von Clarmann et al., 2003; Hoffmann et al., 2005; Carlotti et al., 2006; Dudhia, 2012b). These retrievals exploit the redundancy in MIPAS measurements, which allows the selection of subsets of the spectra, known as microwindows. These microwindows maximise the information content as well as minimise the contribution of other parameters that influence the spectral lines selected, such as spectrally overlapping species, nonLTE emissions, etc.
The use of microwindows instead of broader spectral intervals helps to reduce the size of the matrices involved in the inverse problem as well as the computing cost of the forward model. Nevertheless, when retrieving at mesospheric heights (using special viewing modes) the number of additional microwindows needed to counteract the low $\mathrm{S} / \mathrm{N}$ ratio increases the computing time of the retrieval considerably.

In this study the linear retrievals are compared against the MORSE (MIPAS Oxford Retrieval using Sequential Estimation) results. The MORSE inversion strategy is based on an optimal estimation technique (Rodgers, 2000). It was developed at Oxford University to replicate and if possible improve the results of the standard ESA processor. It has been upgraded to retrieve more constituents as well as to retrieve MIPAS data acquired using different viewing modes (MA mode and UA mode) (Dudhia, 2012b).

\section{2 $p T$ practical considerations}

\subsubsection{Emission from other gases and nonLTE emissions}

Due to the high spectral resolution of MIPAS, the signal contributions of interfering species can be masked out of the measurements by simply removing the measurement points influenced by their emission. The spectral profiles assumed to be due to "pure" $\mathrm{CO}_{2}$ emissions were selected, tangent height at a time, using

$0.9 \leq \frac{L_{\mathrm{CO}_{2}}}{L_{\mathrm{TOT}}} \leq 1.1$,

where $L_{\mathrm{CO}_{2}}$ is a $\mathrm{CO}_{2}$-only simulated spectrum and $L_{\mathrm{TOT}}$ is a simulated spectrum including other gases.

In a similar manner the $\mathrm{CO}_{2}$ spectral points affected by departures from LTE conditions can be masked out from the measurements. These spectral masks can be constructed using

$0.9 \leq \frac{L_{\text {nonLTE }}}{L_{\mathrm{LTE}}} \leq 1.1$,

where $L_{\text {nonLTE }}$ and $L_{\mathrm{LTE}}$ are $\mathrm{CO}_{2}$-only simulated spectra either in nonLTE or in LTE, respectively. The combination of these two masks disregards around $90 \%$ of the spectral points available - around upper mesospheric heights due to 
a combination of emission from other gases and nonLTE effects, and lower down only due to emission from other gases. The $10 \%$ range (i.e. the assumed limits of 0.9 and 1.1) is a compromise between the spectral points available and the influence of the overlapping species and nonLTE emissions. Simulated retrievals showed that this $10 \%$ range removes within $0.3 \mathrm{~K}$ the influence of overlapping species and reduces by around $70 \%$ the impact of nonLTE emissions. To properly take into account the nonLTE emissions, either vibrational temperatures need to be retrieved as part of the retrieval scheme (too computationally expensive) or a vibrational temperature climatology needs to be developed.

\subsubsection{Hydrostatic constraint}

The MIPAS $p T$ retrievals can be constrained using the engineering data defining the line of sight (the pointing information at each tangent height) as an indirect measurement of the state vector. Assuming hydrostatic balance, these engineering altitudes can be used to constrain the temperature and pressure via the hydrostatic equation (Ridolfi et al., 2000)

$z_{i+1}-z_{i}=\left(\frac{R}{g}\right) \frac{T_{i}+T_{i+i}}{2} \log \left(\frac{p_{i}}{p_{i+1}}\right)$,

where $z$ correspond to two tangent profile altitudes ( $i$ and $i+1)$ with associated temperatures $T$ and pressures $p, R$ is the specific gas constant for dry air and $g$ is gravity (as a function of height and latitude).

This constraint is implemented by incorporating the engineering altitudes as part of the measurement vector (with their corresponding uncertainties) and by constructing $p T$ Jacobians differentiating Eq. (19) with respect to either pressure or temperature. This scheme is also used by the operational ESA level 2 retrieval.

\subsubsection{Measurement noise}

One problem of the linear retrieval scheme is that, since it uses entire emission bands rather than just microwindows, the analysis of a single tangent height involves several thousand spectral points. Thus, inverting the measurement error covariance matrix (which in the case of MIPAS is not diagonal due to the correlation of adjacent spectral points due to the apodisation of the spectra) becomes a time-consuming process.

This can be aided by exploiting the almost negligible MIPAS measurement error variation through the day. That is to say, the error covariance matrix can be inverted just once each day allowing for the minor changes in each scan by

$\mathbf{S}_{y}^{-1}=\mathbf{S}_{y_{0}}^{-1}\left(\frac{\boldsymbol{\epsilon}}{\boldsymbol{\epsilon}_{0}}\right)^{-1}$

where $\mathbf{S}_{y}$ is the updated error covariance matrix, $\mathbf{S}_{y_{0}}$ is the error covariance matrix constructed with the mean error of the first scan $\left(\epsilon_{0}\right)$ and $\boldsymbol{\epsilon}$ is the mean error associated with the current scan.

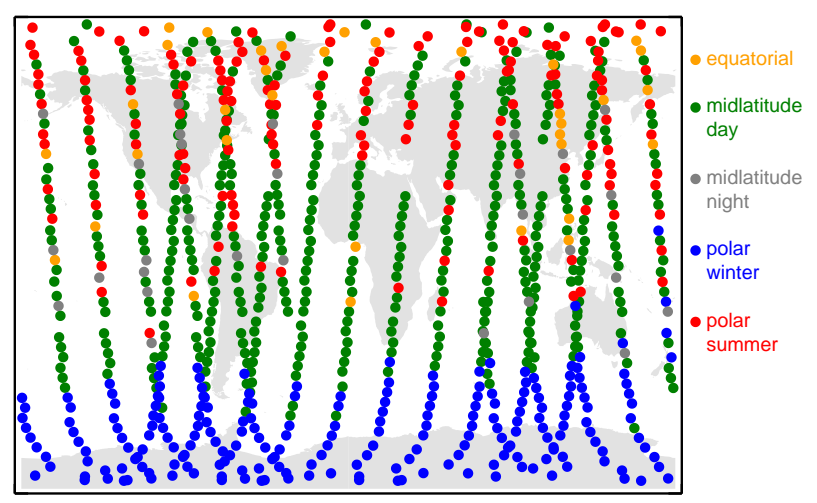

Fig. 15. Linearisation points selected within an ensemble (equatorial, midlatitude day, midlatitude night, polar summer and polar winter profiles) using Eq. (21) for the retrieval of 7 June 2007.

\subsection{Multiple linearisation points}

Despite the linear regime summarised in Sect. 5, there will be always regions where a single linearisation point will be unlikely to be adequate (like the polar vortex). In such cases, several linearisation points may be used to cover more atmospheric variability and the one that minimises a $\chi^{2}$ function (i.e. the square of the residuals between the measurements and the expected spectrum for the linearly adjusted profile) is selected as the solution.

The $\chi^{2}$ function suggested for this purpose is

$\chi^{2}=\frac{[\Delta y]^{T}[\Delta \boldsymbol{y}]}{\boldsymbol{y}^{T} \boldsymbol{y}}$,

where $\Delta \boldsymbol{y}$ is given by

$\Delta \boldsymbol{y}=\boldsymbol{y}-\left[\mathbf{F}\left(\boldsymbol{x}_{0}\right)+\mathbf{K}\left(\boldsymbol{x}-\boldsymbol{x}_{0}\right)\right]$

and the $\boldsymbol{y}^{T} \boldsymbol{y}$ term is needed to weight the residuals according to each tangent height radiance strength.

Figure 15 shows the linearisation points selected for the $p T$ retrieval (from MIPAS spectra band A) of 7 June 2007 using Eq. (21). This linearisation point ensemble consists of five profiles corresponding to equator, midlatitude day, midlatitude night, polar summer and polar winter conditions.

As can be expected, this criterion is selecting for the South Pole (the winter pole in June) mostly the polar winter linearisation profile, while for the North Pole (the summer pole in June) mostly the polar summer linearisation profile. For equatorial and midlatitude conditions, this criterion is selecting a mixture of the day, night midlatitude and the equatorial profiles. This ensemble of linearisation points will be used to gain further insight into the performance of a linear retrieval. Although the equatorial profile is not selected as often as expected, this will not affect the retrieval results as shown in Sect. 7.4.

Typical averaging kernels for the $p T$ linear retrieval are shown in Fig. 16. They describe how the true atmospheric 


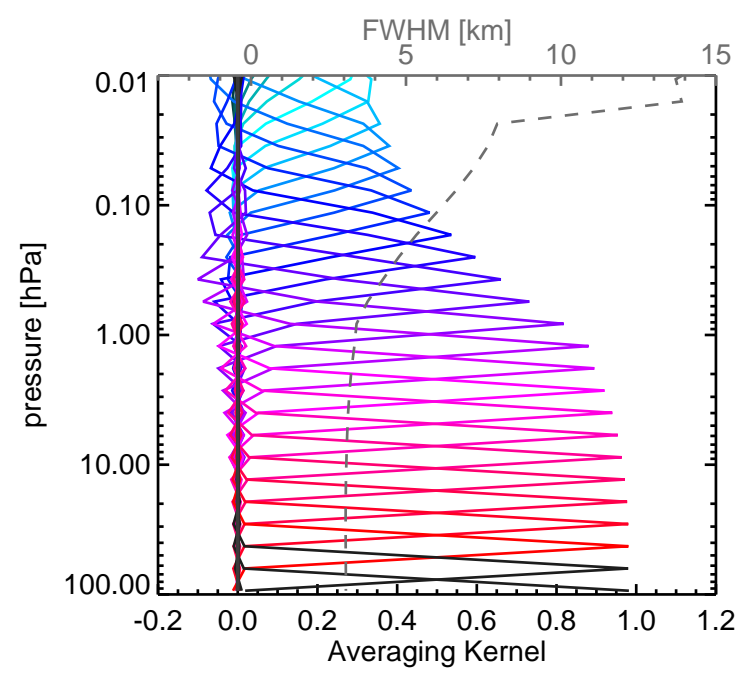

Fig. 16. Averaging kernels for the $p T$ midlatitude day linearisation point (equatorial, midlatitude night, polar summer and polar winter are very similar).The dashed grey line is a measure of the vertical resolution of the retrieved profile (derived from the full width at half maximum (FWHM) of the averaging kernels).

state has been distorted by the retrieval at each pressure level. A measure of the vertical resolution of the retrieval is given by the half width of each kernel. As shown, the $p T$ retrieval has a vertical resolution of about $3 \mathrm{~km}$ in the stratosphere, increasing up to $8 \mathrm{~km}$ in the lower mesosphere and up to $14 \mathrm{~km}$ in the upper mesosphere.

\subsection{Results}

To gain an idea of the performance of the linear retrieval, Fig. 17 shows a zonal mean comparison for 7 June 2007 between the linear retrieval, MORSE and Microwave Limb Sounder (MLS) (Waters et al., 1999, 2006) dataset. It also displays the COSPAR International Reference Atmosphere (CIRA, 1988) climatology for that period - not to be confused with the linearisation points used (shown in Fig. 15).

MLS is an instrument that measures thermal microwave limb emission in five spectral regions from $118 \mathrm{GHz}$ to 2.5 Thz. It was launched in July 2004 on board the Aura spacecraft. MLS scans the limb from the ground to about $95 \mathrm{~km} 3500$ times per day. It covers between $82^{\circ} \mathrm{S}$ and $82^{\circ} \mathrm{N}$, providing near global coverage. The vertical resolution of this data is about $3 \mathrm{~km}$ (Waters et al., 1999, 2006). Its temperature retrievals have been extensively validated (Schwartz et al., 2008).

The linear retrieval results display the structure shown in the climatology data, in MORSE and in MLS with cold temperatures in the polar summer mesopause $\left(>50^{\circ} \mathrm{N}\right.$ for this day) and a higher stratopause in the polar winter $\left(<50^{\circ} \mathrm{S}\right)$. However, as seen in the absolute difference subplot, compared to MORSE and MLS the linear retrieval seems to overestimate the temperatures for pressures between 0.1 and $0.01 \mathrm{hPa}$ at all latitudes and underestimate the temperature for pressures between 1 and $0.1 \mathrm{hPa}$. For pressures greater than $1 \mathrm{hPa}$ in general there seems to be no significant difference (less than $3 \mathrm{~K}$ ) between the linear, MORSE and the MLS results.

Figure 18 shows a profile by profile seasonal comparison between the linear retrieval, MORSE and MLS data. For the linear-MLS comparisons, two profiles were considered to be coincident if they were within $500 \mathrm{~km}$ radius and $6 \mathrm{~h}$. The seasonal bins compared correspond to latitude bins between $90^{\circ} \mathrm{S}-50^{\circ} \mathrm{S}, 20^{\circ} \mathrm{S}-20^{\circ} \mathrm{N}$ and $50^{\circ} \mathrm{N}-90^{\circ} \mathrm{N}$ and sorted into winter, equatorial and summer according to the date of the profile. These bins were selected to cover most of the atmospheric variability.

As shown in Fig. 18, there seems to be an overestimation of the temperature by the linear retrieval for pressures lower than around $0.1 \mathrm{hPa}$. This overestimation varies with season and it is around $3-5 \mathrm{~K}$ for the MORSE comparisons and up to around $5-10 \mathrm{~K}$ for MLS ones. For greater pressures the bias found is less than $3 \mathrm{~K}$.

In addition to the mean bias, this figure also show the standard deviation of the profiles compared to gain an idea of the variability of the data. For pressures greater than $0.5 \mathrm{hPa}$, the variability found in most seasonal bins (for the MORSE and MLS comparisons) is approximately $3 \mathrm{~K}$, which is the expected error margin for a $10 \mathrm{~K} \boldsymbol{x}-\boldsymbol{x}_{0}$ "distance".

These comparisons might suggest that the linear retrieval is not as precise as needed for pressures lower than $0.1 \mathrm{hPa}$. However, it should be pointed out that the performance of the linear retrieval will improve when a proper linearisation point climatology is used. Even if when using this climatology the retrievals are not as precise as needed, the linear results are presumably going to be closer to the true state than the climatology, so they may be used as a first iteration in the iterative schemes, potentially saving computing time.

\subsection{Further VMR practical considerations}

As for the $p T$ retrievals, the VMR retrievals will be influenced by overlapping emissions of other gases and by nonLTE processes. These influences can be reduced using spectral masks as the ones described in section 7.2.1. In this case, the combination of these two masks disregards around $88 \%$ of the spectral points available, mainly due to emission from other gases.

The linearly retrieved VMR altitude range was truncated above the altitude where the estimated random error was greater than $30 \%$. Above these altitudes the retrieved values are dominated by noise. Although coaddition could be used to retrieve gas concentrations at upper mesospheric altitudes, this approach is not pursued here. The vertical resolution of these retrievals is $3 \mathrm{~km}$ throughout the entire vertical retrieval range. 

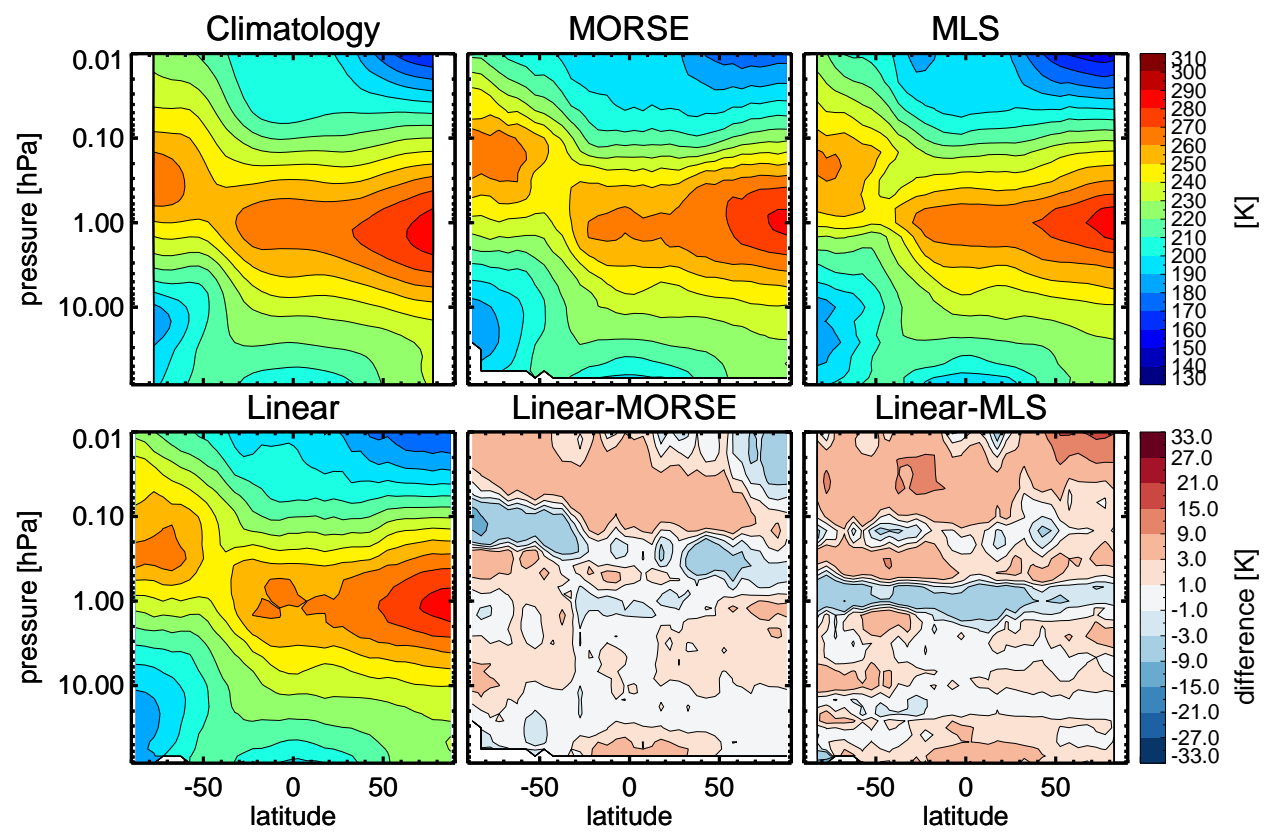

Fig. 17. Temperature zonal mean for 7 June 2007 for climatology, MORSE, MLS and the MIPAS linear retrieval. The top row displays climatology, MORSE and MLS, while the bottom row displays the MIPAS linear retrieval results as well as the absolute difference between the retrievals (linear-MORSE and linear-MLS). Note that the absolute difference contours are not equally spaced.
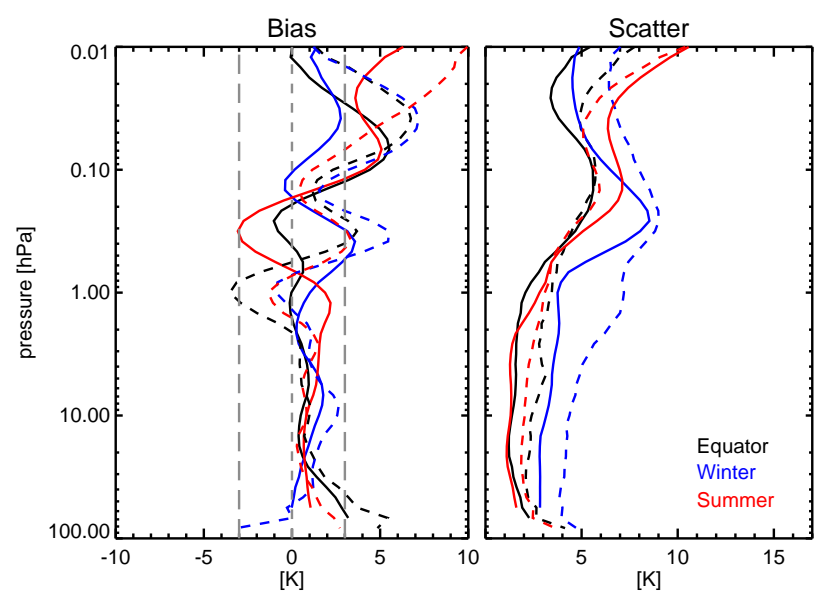

Fig. 18. Mean temperature biases (left) and scatter (right) between the MIPAS linear retrieval, MORSE (solid) and MLS (dashed). The grey dashed line shows a $3 \mathrm{~K}$ difference. The number of profiles compared in each bin for the MLS comparison were 40, 2918 and 3159 for the equatorial, winter and summer case, respectively.

Currently, for each $p T$ linearisation point there is one $\mathrm{CH}_{4}$ profile associated with it. However, it should be noted that, tentatively, for each $p T$ linearisation point, different $\mathrm{CH}_{4}$ scenarios can be simulated. At each scan, a retrieval will be performed for each scenario and the one that best minimises the $\chi^{2}$ suggested in Sect. 7.3 (Eq. 21) used as solution.

\subsection{VMR results}

Figure 19 displays the zonal mean $\mathrm{CH}_{4}$ comparison between the linear retrieval and MORSE for 7 June 2007. It also displays the IG climatology (Remedios et al., 2007) for that period, not to be confused with the linearisation points used (displayed in Fig. 15). As shown, the linear retrieval displays the structure found in the climatology and in MORSE with higher values around the equator; however, as seen in the percentage difference subplot, the linear retrieval seems to overestimate the $\mathrm{CH}_{4}$ concentration by around $20 \%$ for pressures between 10 and $1 \mathrm{hPa}$.

Figure 20 displays a profile-by-profile comparison between the linear retrieval and MORSE for the same days as in Sect. 7.4. As can be seen the linear retrieval is always overestimating the $\mathrm{CH}_{4}$ concentrations. Figure 21 shows VMR, pressure and temperature zonal mean $\boldsymbol{x}-\boldsymbol{x}_{0}$ "distances", or in other words the separation between the retrieved atmospheric state and the linearisation points used. For most altitudes and latitudes, these "distances" are greater than the requirements to fall within the linear regime (see Sect. 4), suggesting that the linear retrieval results should improve once a more reasonable linearisation point climatology is used.

In the case where the linearisation point is not a good representative of the atmospheric state, the linear retrieval results may be used as a first iteration in an iterative scheme, or if the linear retrieval error is greater than the expected climatological variation, the iterative scheme can be used starting from the best initial guess available. 

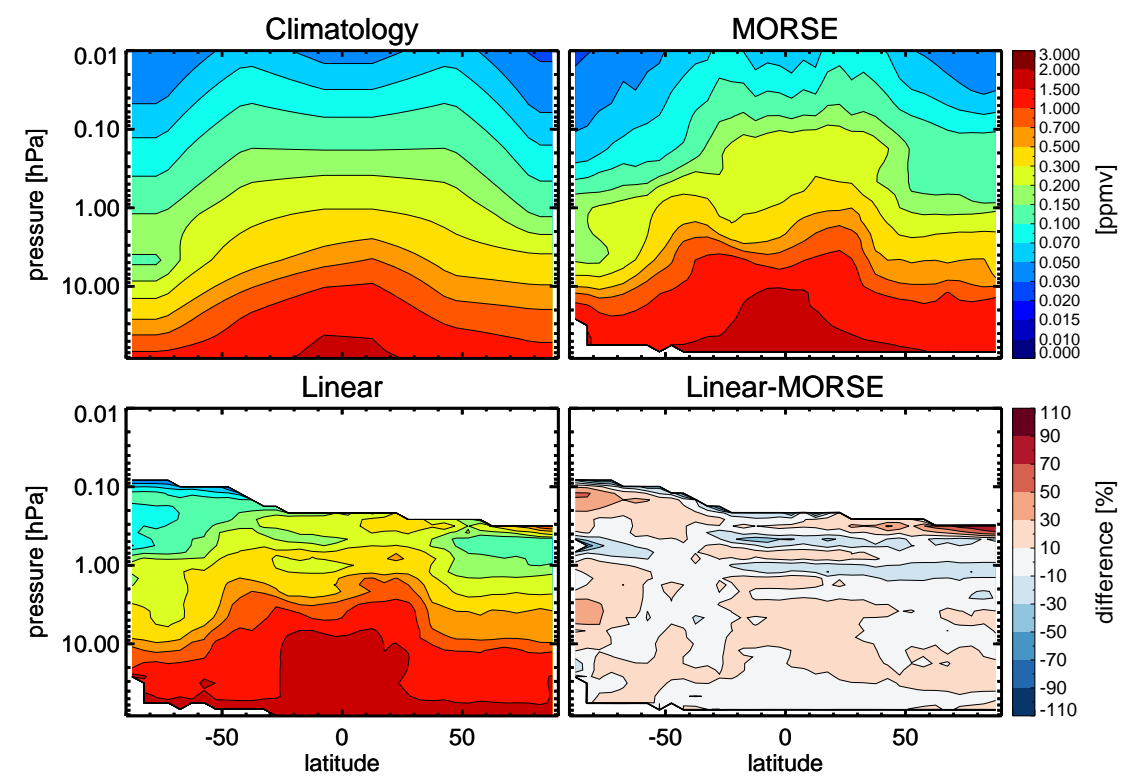

Fig. 19. $\mathrm{CH}_{4}$ zonal mean for 7 June 2007 for climatology, MORSE and the MIPAS linear retrieval. The top row displays climatology and MORSE, while the bottom row displays the MIPAS linear retrieval results as well as the percentage difference between the two retrievals (linear-MORSE).
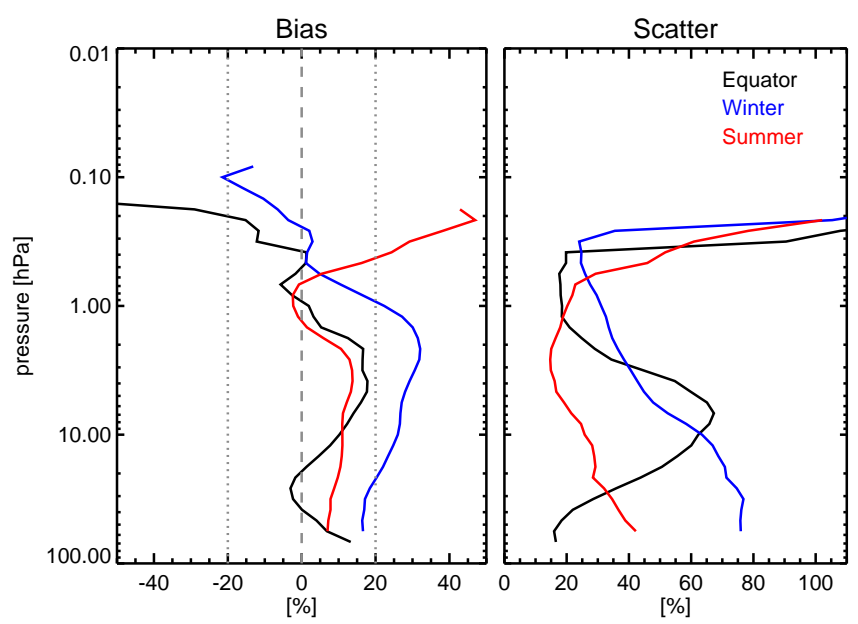

Fig. 20. Mean $\mathrm{CH}_{4}$ bias (left) and scatter (right) between the MIPAS linear retrieval and MORSE. The grey dotted line shows the expected equatorial $\mathrm{CH}_{4}$ variability. The grey dashed line shows a $3 \mathrm{~K}$ difference.

Since $\mathrm{CH}_{4}$ does not possess pure rotational bands and hence no microwave signature in the spectrum, a comparison against MLS is not possible. A comparison against other instruments was considered unnecessary at this stage due to the clear overestimation (considering the differences against the IG climatology and MORSE) of the linear $\mathrm{CH}_{4}$ retrievals.

\section{Retrieval speed}

An important feature of the linear retrieval is its speed. Since there is no need to run the radiative transfer model, once the error covariance matrices are inverted (only needed once a day), the main time-consuming processes are the assimilation of the data into the retrieval and the inversion of the multiple linearisation points (five in the current configuration of the scheme). Using the same computer to run MORSE and the linear retrieval, it has been determined that the linear retrieval is faster than MORSE at least by a factor of 20 (reducing the processing time of a day of middle atmosphere data from days to a couple of hours on a single CPU) when retrieving $p T$ and $\mathrm{CH}_{4}$. Furthermore, it should be noted that speed of the linear retrieval can be improved by retrieving only some of the linearisation points, selecting them according to the time and latitude of the scene.

\section{Conclusions}

An alternative algorithm to retrieve temperature, pressure and composition from limb-viewing infrared spectrometers has been introduced. This algorithm performs the inversion without rerunning the radiative transfer model as long as the linearisation point is close enough to the scene conditions. This new algorithm can use the whole spectral band of the molecule rather than a small subset of it (the microwindows) and therefore, theoretically, leads to a higher precision.

It was shown that the retrievals of pressure and temperature can be treated linearly up to a $20 \% \boldsymbol{x}-\boldsymbol{x}_{0}$ "pressure 

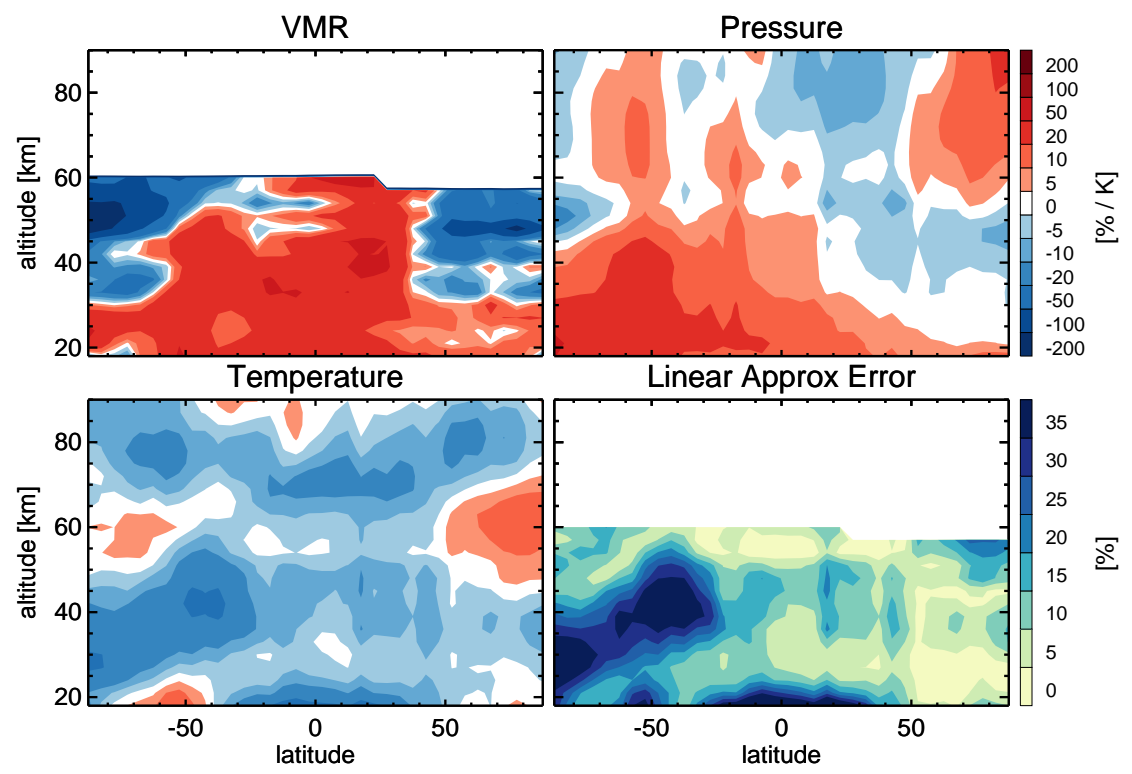

Fig. 21. VMR, pressure and temperature $\boldsymbol{x}-\boldsymbol{x}_{0}$ "distances" (in percentage or Kelvin) for 7 June 2007. Note that the "distance" contours are not equally spaced. Also (bottom right corner), the $\mathrm{CH}_{4}$ linear approximation error, computed using equation 16, is shown.

distance" for a $3 \%$ error margin and up to $10 \mathrm{~K}$ "distance" for a $3 \mathrm{~K}$ error margin near the stratopause and less than $0.5 \mathrm{~K}$ elsewhere.

Assuming perfect $p T$ knowledge, it was shown that the $\mathrm{CH}_{4}$ concentrations can be treated linearly up to a $\boldsymbol{x}-\boldsymbol{x}_{0}$ "distance" of $20 \% \mathrm{CH}_{4}$ concentration for a $2 \%$ error margin. However, since the linear algorithm requires precomputed simulated spectra and Jacobians for the $p T$ and $\mathrm{CH}_{4}$ conditions of the linearisation points, adjustments for the $p T$ dependence of the simulated spectra and Jacobians have been proposed. These $\mathrm{CH}_{4} p T$ adjustments hold up to a $10 \mathrm{~K}$ and $20 \%$ pressure $\boldsymbol{x}-\boldsymbol{x}_{0}$ "distance" with an error margin of $20 \%$ and $10 \%$, respectively.

In order to fulfill all these restrictions (i.e. the required proximity between the linearisation point and the true profile) a $p T$ and $\mathrm{CH}_{4}$ climatology with a latitudinal resolution of at least $20^{\circ}$ of latitude is suggested. Furthermore, it was also shown how to select the best retrievals from different linearisation points for regions where a single linearisation point is unlikely to be adequate.

This retrieval scheme was implemented using MIPAS radiances and compared to the results of the MIPAS iterative scheme MORSE and the results of MLS.

The $p T$ comparisons revealed that linear retrieval is within a $3 \mathrm{~K}$ range difference on average for pressures greater than $0.1 \mathrm{hPa}$, while for lower pressures there seems to be an overestimation of the temperature by the linear scheme up to $5 \mathrm{~K}$. The $\mathrm{CH}_{4}$ comparisons showed that the linear retrieval is overestimating the $\mathrm{CH}_{4}$ concentrations. This overestimation may be due to the lack of proper linearisation points climatology, especially considering the $\mathrm{CH}_{4}$ retrieval temperature requirements to fall within the linear regime.

Even if the linear retrieval is not as precise as required, it has proven its potential to be close to the actual scene conditions that they may be used as the first iteration of the iterative schemes, thereby reducing the processing time.

\section{Appendix A}

\section{Linearising the forward model}

The radiance $I_{\nu}$ measured by an instrument is given by

$I_{\nu}=\int_{\nu} \int_{\omega} F_{\nu} \mathrm{d} \nu * \Phi \mathrm{d} \omega * L_{\nu}$,

where $v$ is wavenumber, $\omega$ is solid angle, $F_{v}$ is the instrument spectral response, $\Phi$ is the field of view and $L_{v}$ is the radiance that reaches the satellite given by the radiative transfer equation

$L_{v}=L_{0 v} \tau_{v}(x)+\int_{0}^{x} B_{v}(T) \frac{\mathrm{d}}{\mathrm{d} x^{\prime}}\left(\tau_{v}\left(x^{\prime}\right)\right) \mathrm{d} x^{\prime}$,

where $x$ is distance along the path, $L_{0 v}$ represents the background emission at the end of the path (negligible for the infrared region of the spectrum), $B_{v}(T)$ is the Planck function, which describes the thermal emission of the atmosphere (assuming local thermodynamic equilibrium and neglecting scattering) and $\tau_{v}$ is the transmittance of the path from the point $x^{\prime}$ to the spacecraft $(x=0)$. This quantity is defined by 


\section{References}

$\tau_{v}(x)=\exp \left(-\chi\left(x_{v}\right)\right)$

and

$$
\chi_{v}(x)=\int_{0}^{x} \sum_{j} v\left(x^{\prime}\right) \sigma_{v}\left(x^{\prime}\right) \rho\left(x^{\prime}\right) \mathrm{d} x^{\prime},
$$

where $\chi_{v}$ is the optical thickness of the path, $j$ corresponds to the $j$ th absorber with absorption coefficient $\sigma_{v}$, volume mixing ratio $v$ and density $\rho$.

Assuming a stratified atmosphere, the numerical integration of Eqs. (A2) and (A3) can be formulated as

$$
L_{v}=L_{0 v} \tau_{0 v}+\sum_{i} B_{v}\left(T_{i}\right)\left[\tau_{v(i-1)}-\tau_{v(i)}\right]
$$

and

$$
\tau_{v}=\exp \left(-\sum_{i} \sum_{j} \rho_{i} l_{i} v_{i j} \sigma_{i j}\right),
$$

where $i$ refers to the $i$ th stratified level, $\tau_{i}$ corresponds to the transmittance from the satellite up the given stratified level and $l_{i}$ is the length along the path in that level.

Assuming that the path is optically thin $(\chi \ll 1)$, it can be shown that Eq. (A6) can be approximated by

$$
L_{v} \simeq \sum_{i} B_{v}\left(T_{i}\right) \rho_{i} l_{i}\left(\sum_{j} v_{i j} \sigma_{v(i j)}\right)
$$

and using the ideal gas law by

$L_{v} \simeq \sum_{i} B_{v}\left(T_{i}\right) \frac{p_{i}}{R T_{i}} l_{i}\left(\sum_{j} v_{i j} \sigma_{v(i j)}\right)$,

which is the "most" linear scenario of the radiative transfer (except in the microwave region of the spectra where $B_{v}(T) \simeq T$, and hence much of the temperature dependence cancels) and still involves the product of atmospheric variations depending on $T, p, v$ and $\sigma_{\nu}$.

However, assuming that the spectral sampling of the instrument $\left(\simeq 0.5 \mathrm{~cm}^{-1}\right)$ is coarse relative to the actual line width $\left(\simeq 0.001 \mathrm{~cm}^{-1}\right.$ in the upper stratosphere) we can assume $\sigma_{v}$ independent of $p, T$ or $v$. Under this scenario, Eq. (8) implies that when the path is optically thin, the forward model is linear with respect to $p, v$ and $B_{v}(T) / T$.

Acknowledgements. This work was part of a DPhil undertaken at the University of Oxford under the funding of the National Council on Science and Technology of Mexico (CONACYT). Part of this research was carried out at the Jet Propulsion Laboratory, California Institute of Technology, under a contract with the National Aeronautics and Space Administration.

Edited by: H. Worden
Bernath, P. F.: Atmospheric chemistry experiment (ACE): Analytical chemistry from orbit, Trends Analyt. Chem., 25, 647-654, 2006.

Carlotti, M., Brizzi, G., Papandrea, E., Prevedelli, M., Ridolfi, M., Dinelli, B., and Magnani, L.: GMTR: Two-dimensional geofit multitarget retrieval model for Michelson Interferometer for Passive Atmospheric Sounding/Environmental Satellite observations, Appl. Optics, 45, 4,doi.org/10.1364/AO.45.000716, 2006.

CIRA.: CIRA Part I: Thermosphere Model, Adv. Space Res., 8, 56, 1988.

Dudhia, A.: Microwindow selection for the MIPAS reduce resolution mode, Proceedings of the third workshop on the Atmospheric Chemistry Validation of Envisat (ACVE-3), 2007.

Dudhia, A.: Reference Forward Model software manual, available at: http://www.atm.ox.ac.uk/RFM/ (last access: 23 April 2013), 2012a.

Dudhia, A.: MORSE: MIPAS optimal retrieval using sequential estimation, available at: http://www.atm.ox.ac.uk/MORSE/ (last access: 23 April 2013), 2012b.

Dudhia, A., Jay, V., and Rodgers, C.: Microwindow selection for high spectral resolution sounders, Appl. Optics, 41, 3665-3673, doi:10.1364/AO.41.003665, 2002.

Endemann M.: MIPAS instrument Concept and Performance, ESAESTEC, 1999.

Fischer, H., Blom, C., Oelhaf, H., Carli, B., Carlotti, M., Delbouille, L., Ehhalt, D., Flaud, J., Isaksen, I., Lopez-Puertas, M., McElroy, C., and Zander, R.: Envisat-MIPAS, the Michelson Interferometer for Passive Atmospheric Sounding; An instrument for atmospheric chemistry and climate research, SP-1229, 2000.

Fischer, H., Birk, M., Blom, C., Carli, B., Carlotti, M., von Clarmann, T., Delbouille, L., Dudhia, A., Ehhalt, D., Endemann, M., Flaud, J. M., Gessner, R., Kleinert, A., Koopman, R., Langen, J., López-Puertas, M., Mosner, P., Nett, H., Oelhaf, H., Perron, G., Remedios, J., Ridolfi, M., Stiller, G., and Zander, R.: MIPAS: an instrument for atmospheric and climate research, Atmos. Chem. Phys., 8, 2151-2188, doi:10.5194/acp-8-2151-2008, 2008.

Gille, J. and House, F.: On the Inversion of Limb Radiance Measurements I: Temperature and Thickness, J. Atmos. Sci., 28, 1427-1442, 1971.

Hoffmann, L., Spang, R., Kaufmann, M., and Riese, M.: Retrieval of CFC-11 and CFC-12 from Envisat MIPAS observations by means of rapid radiative transfer calculations, Adv. Space Res., 36, 915-921, doi:10.1016/j.asr.2005.03.112, 2005.

López-Puertas, M. and Taylor, F. W.: Non-LTE Radiative Transfer in the Atmopshere, Vol. 3 of Series on Atmospheric, Oceanic and Planetary Physics, World Scientific, 2001.

Remedios, J., Leigh, R., Sembhi, H., and Waterfall, A.: New IG2 seasonal climatologies for MIPAS, Proceedings Envisat Symposium, 2007.

Ridolfi, M., Carli, B., Carlotti, M., von Clarmann, T., Dinelli, B., Dudhia, A., Flaud, J.-M., Hopfner, M., Morris, P., Raspollini, P., Stiller, G., and Wells, R.: Optimised forward model and retrieval scheme for MIPAS near-real time and data processing, Appl. Optics 39, 1323-1340, doi:10.1364/AO.39.001323, 2000.

Rodgers, C.: Inverse Methods for Atmospheric Sounding: Theory and Practice, Vol. 2 of Series on Atmospheric, Oceanic and Planetary Physics, World Scientific, 2000. 
Schwartz, M., Lambert, A., Manney, G., Read, W., Livesey, N., Froidevaux, L., Ao, C., Bernath, P., Boone, C., Cofield, R., Daffer, W., Drouin, B., Fetzer, E., Fuller, R., Jarnot, R., Jiang, J., Jiang, Y., Knosp, B., Kruger, K., Li, J.-L., Mlynczak, M., Pawson, S., III, J. R., Santee, M., Snyder, W., Stek, P., Thurstans, R., Tompkins, A., Wagner, P., Walker, K., Waters, J. and Wu, D.: Validation of the Aura Microwave Limb Sounder Temperature and Geopotential Height Measurements, J. Geophys. Res. 113, D15S11, doi:10.1029/2007JD008783, 2008.

Tikhonov, S.: On the solution of incorrectly stated problems and a method of a regularization, Dokl. Acad. Nauk. SSSR, 501-504, 1963.

Twomey, S.: On the numerical solution of Fredholm integral equation of the first kind by the inversion of linear system produced by quadrature, J. Asss. Comput. Mach, 10, 97-101, 1963.

von Clarmann, T., Glatthor, N., Grabowski, U., Höpfner, M., Kellmann, S., Kiefer, M., Linden, A., Mengistu Tsidu, G., Milz, M., Steck, T., Stiller, G. P., Wang, D. Y., Fischer, H., Funke, B., Gil-López, S., and López-Puertas, M.: Retrieval of temperature and tangent altitude ponting from limb emission spectra recorded from space by the Michelson Interferometer for Passive Atmospheric Sounding (MIPAS), J. Geophys. Res., 108, 4736, doi:10.1029/2003JD003602, 2003.
Waters, J., Read, W., Froidevaux, L., Jarnot, R., Cofield, R., Flower, D., Lau, G., Pickett, H., Santee, M., Wu, D., Boyles, M., Burke, J., Lay, R., Loo, M., Livesey, N., Lungu, T., Manney, G., Nakamura, L., Perun, V., Ridenoure, B., Shippony, Z., Siegel, P., Thurstans, R., Harwood, R., and Filipiak, M.: The UARS and EOS Microwave Limb Sounder Experiments, J. Atmos. Sci. 56, 194-218, 1999.

Waters, J., Froidevaux, L., Harwood, R., Jarnot, R., Pickett, H., Read, W., Siegel, P., Cofield, R., Filipiak, M., Flower, D., Holden, J., Lau, G., Livesey, N., Manney, G., Pumphrey, H., Santee, M., Wu, D., Cuddy, D., Lay, R., Loo, M., Perun, V., Schwartz, M., Stek, P., Thurstans, R., Boyles, M., Chandra, S., Chavez, M., Chen, G.-S., Chudasama, B., Dodge, R., Fuller, R., Girard, M., Jiang, J., Jiang, Y., Knosp, B., LaBelle, R., Lam, J., Lee, K., Miller, D., Oswald, J., Patel, N., Pukala, D., Quintero, O., Scaff, D., Snyder, W., Tope, M., Wagner, P., and Walch, M.: The Earth Observing System Microwave Limb Sounder (EOS MLS) on the Aura satellite, IEEE Trans. Geosci. Remote Sens., 44, 1075-1092, doi:10.1109/TGRS.2006.873771, 2006. 